\title{
Réponses inflammatoires au cours d'une série de matchs chez des handballeurs de l'élite en République du Bénin
}

\section{L.S.O. LINTA ${ }^{1 *}$, P. GOUTHON ${ }^{1}$, T. HOUNTOHOTEGBE ${ }^{2}$, F. MESSAN ${ }^{3}$, B.K. NOUATIN ${ }^{1}$, J.P. KOUASSI et A. BIGOT ${ }^{2}$}

\author{
${ }^{1}$ Unité de Recherche Sport, Santé et Evaluation, (UR/SSE), Institut National de la Jeunesse, de l'Education \\ Physique et du Sport. Université d'Abomey-Calavi, Porto-Novo, République du Bénin. \\ ${ }^{2}$ Laboratoire d'immunogénétique et inflammation, Faculté des Sciences de la Santé. Université d'Abomey- \\ Calavi, Cotonou, République du Bénin. \\ ${ }^{3}$ Unité d'Explorations Respiratoire, Hormonale et Gérontologique du Sportif, Institut National de la Jeunesse, \\ de l'Education Physique et du Sport. Université d'Abomey-Calavi, Porto-Novo, République du Bénin. \\ *Auteur correspondant ; E-mail : lintalonce@yahoo.fr; Tél : (+229) 97586906.
}

\section{RESUME}

Les données liées aux réponses inflammatoires en situation de match chez les handballeurs qui pratiquent dans un environnement immunostimulant comme celui du Bénin, ne sont pas disponibles. Cette étude avait pour objectif de déterminer chez des handballeurs appartenant à l'élite du Bénin, les modifications des paramètres associés à la réponse inflammatoire, induites par une série de trois matchs expérimentaux. Il s'agit d'une étude d'intervention, entreprise avec 16 joueurs juniors (JJ) et seniors (JS), répartis de façon aléatoire en deux équipes $\mathrm{A}$ et $\mathrm{B}$, qui se sont affrontées pendant ces matchs. La numération des leucocytes circulants a été déterminée, les interleukines Il-6, Il-10 et le tumor necrosis factor (TNF- $\alpha$ ) ont été dosés avant, juste à la fin, puis 24 heures après le premier (Match 1) et le troisième (Match 3) matchs. Les accroissements des moyennes des leucocytes totaux, des neutrophiles, de l'IL-6, de l'IL-10 et du TNF- $\alpha$ enregistrés au cours du troisième match étaient plus importants $(\mathrm{p}<0,05)$ que ceux observés à la fin du premier. Après 24 heures de récupération, la restauration des lymphocytes était de $83,3 \%$, celle des leucocytes totaux de 76,6\%, celle des neutrophiles de 57,7\%, celles du TNF- $\alpha$, de l'IL-6, de l'IL-10, respectivement de 61,3\%, 64,0\% et 57,2\%. La série de trois matchs a eu un effet cumulatif sur l'augmentation des moyennes des paramètres étudiés et la récupération $24 \mathrm{~h}$ après le dernier match n'a atteint $100 \%$ pour aucun d'eux. Il est donc impérieux que des travaux soient réalisés à brève échéance, pour déterminer la méthode d'entraînement qui induit à la fois le développement des capacités physiques et la récupération rapide du système immunitaire en période d'entraînement et de compétition chez ces joueurs.

(C) 2020 International Formulae Group. All rights reserved.

Mots clés : Leucocytes, cytokines, compétition, Afrique subsaharienne.

\section{Inflammatory responses during a series of matches among elite handball players in Benin}

\begin{abstract}
Data related to inflammatory responses in match situations among handball players who practice in an immunostimulating environment such as the one in the Republic of Benin are not available. The aim of this study was to determine among elite male handball players, the changes in the parameters associated with the
\end{abstract}


inflammatory response, induced by a series of three experimental matches. This research was an intervention study, undertaken with 16 junior (JP) and senior (SP) players, randomly assigned to two teams A and B for the experimental matches. Circulating leukocyte counts were determined, interleukins Il-6, Il-10, and tumor necrosis factor (TNF- $\alpha$ ) were measured before, just at the end, and 24 hours after the first (Match 1) and the third (Match 3) matches. The increases in the averages of total leukocyte, neutrophil counts, the IL-6, IL-10 and TNF- $\alpha$ concentrations recorded during the third match were greater $(p<0.05)$ than those observed at the end of the first. After 24 hours from the end, lymphocyte recovery was $83.3 \%$, total leukocyte counts recovery was $76.6 \%$, that of neutrophils was $57.7 \%$. The TNF- $\alpha$, IL-6 and IL-10 concentrations were respectively restored at $61.3 \%, 64.0 \%$, and $57.2 \%$. The three-match series had a cumulative effect on the increase in the mean values of the parameters studied. The 24 hours recovery following the last match was insufficient for $100 \%$ restoration of the inflammatory response makers. Therefore, it is imperative to carry out studies to determine a training method that induces both development of physical capacities and rapid recovery of the immune system during training sessions' and competitions' in these players.

(C) 2020 International Formulae Group. All rights reserved.

Keywords: Leukocytes, cytokines, competition, sub-Saharan Africa.

\section{INTRODUCTION}

$\mathrm{Au}$ handball, les matchs sont caractérisés par des actions d'intensités élevées telles que les courses à vitesse maximale, les sauts, les tirs, les changements rapides de direction (Hermassi et al., 2018). Les matchs joués à des intensités beaucoup plus élevées que celles auxquelles le joueur était habitué, peuvent avoir comme conséquence, l'altération des fonctions de l'organisme, dont celle immunitaire. Cette altération se traduit souvent par une immunodépression, conduisant à une augmentation du risque d'infection pendant 72 heures (Nielsen et al., 2016). Les matchs induisent des réponses inflammatoires visant, la réparation des dommages musculaires et la restauration de la fonction des cellules immunocompétentes (Noack et al., 2018; Souglis et al., 2018).

Après le match, le temps mis pour la réparation des tissus lésés et la restauration de la fonction immunitaire doit être considéré comme une période de récupération dont la durée dépend entre autres, de l'intensité à laquelle les athlètes ont joué (CuryBoaventura et al., 2018). La récupération de la fonction immunitaire après une séance d'exercice physique intense chez l'athlète est d'une importance capitale, puisque le sport de haut-niveau est actuellement caractérisé par des séances d'entraînement biquotidiennes et un enchaînement des compétitions au cours de l'année (Bessa et al., 2016).

$\mathrm{Au}$ handball, pour des raisons budgétaires, les compétitions sont de plus en plus organisées sous forme de tournoi groupé, au cours duquel les équipes jouent au moins un match toutes les 24 heures. Elles se déroulent souvent en deux ou plusieurs tours, dont le premier comporte la plupart du temps, trois matchs pour chaque équipe, les tours suivants étant réservés aux meilleures. Chaque match dure deux mi-temps de $30 \mathrm{~min}$, avec 10 ou 15 min de pause (IHF, 2016) avec des actions dont les intensités se situent entre 75 et $85 \%$ de la fréquence cardiaque maximale des joueurs (Póvoas et al., 2012; Nouatin et al., 2015). Dans ces conditions, les équipes jouent lors du premier tour, au moins trois matchs en 72 heures. Le temps de récupération entre les matchs se situe autour de 24 heures et ne dépasse pas 48 heures entre les rencontres d'un tour et celles du tour suivant. Dans ces conditions, ce temps de récupération peut ne pas suffir à la récupération totale de la fonction immunitaire des joueurs. Au cours de telles compétitions, la fonction immunitaire des joueurs pourrait être par conséquent affaiblie, augmentant ainsi le risque d'infection, surtout celle des voies respiratoires supérieures (Nielsen et al., 2016). 
Les effets de l'exercice physique intense sur les paramètres associés à la réponse inflammatoire ont été rapportés par plusieurs études (Cury-Boaventura et al., 2018 ; Souglis et al., 2018). Il est ainsi apparu que, l'exercice physique intense induit une polynucléose neutrophile, une monocytose (Pedersen et Hoffman-Goetz, 2000), une lymphocytose médiée par l'intervention des catécholamines (Cury-Boaventura et al., 2018), une libération accrue des cytokines anti-inflammatoires IL-10, IL-1ra et proinflammatoires TNF- $\alpha, \quad$ IL-6, IL-1 $\beta, \quad$ IL-8 (Harnish et Sabo, 2016; Mohr et al., 2016; Souglis et al., 2018). Immédiatement après l'exercice, la numération leucocytaire change et tend vers les données de repos (Gunzer et al., 2012). La lymphocytose se transforme sous l'effet du cortisol en lymphopénie, avant que les valeurs ne remontent à celles de repos (Cury-Boaventura et al., 2018).

Dans le contexte particulier du Bénin, caractérisé par un environnement chaud et humide (Gouthon et al., 2009), il faudrait s'attendre à des réponses inflammatoires plus accentuées si les handballeurs sont soumis à des compétitions groupées. Le Bénin est en effet, situé en Afrique subsaharienne, dans laquelle les valeurs des températures ambiantes et les pourcentages de l'humidité relatives sont généralement élevés et atteignent très souvent respectivement $35{ }^{\circ} \mathrm{C}$ et $90 \%$ (Asseh et al., 2019; Kouakou et al., 2019). Une séance d'entraînement ou un match joué dans un environnement chaud, induit en effet des réactions inflammatoires plus accentuées, que celles observées dans un climat tempéré (Mitchel et al., 2002).

A notre connaissance, aucune étude ne s'est encore intéressée à l'évaluation de la réponse inflammatoire au cours d'une série de trois matchs consécutifs chez les handballeurs en Afrique subsaharienne. La seule étude réalisée au Bénin sur ce sujet, concerne plutôt l'effet d'une série de matchs sur les performances à des tests de terrain (Nouatin et al., 2015).

Il est donc impérieux que des travaux expérimentaux soient menés, dans le but de mieux comprendre comment se comporte le système immunitaire des joueurs de handball engagés dans une compétition groupée organisée en ambiance chaude et humide. La présente étude visait par conséquent à: 1) comparer chez des handballeurs appartenant à l'élite du Bénin, les modifications des paramètres associés à la réponse inflammatoire au cours du premier d'une série de trois matchs expérimentaux à celles du troisième; 2) mesurer chez les mêmes joueurs, les taux de restauration de ces paramètres, 24 heures après la fin de la série de trois matchs.

\section{MATERIEL ET METHODES}

\section{Protocole de l'étude}

Il s'agit d'une étude d'intervention, réalisée à Porto-Novo sous forme d'une série de trois matchs expérimentaux, avec des handballeurs appartenant tous à l'élite masculine du Bénin. Une semaine avant les matchs, tous les joueurs ont passé le test 30-15 IFT (Buchheit, 2005) pour l'estimation indirecte du $\dot{V} \mathrm{O}_{2} \max$ et la détermination de la fréquence cardiaque maximale. Le jour de chaque match, avant l'échauffement, les mesures anthropométriques ont été prises chez tous les athlètes retenus. Avant, juste à la fin et 24 heures après chaque match, des mesures de la masse corporelle (MC), de la température rectale (Trec) et de la fréquence cardiaque $(\mathrm{Fc})$ ont été effectuées. Le jour des premier et troisième matchs, avant l'échauffement et juste à la fin, puis 24 heures après, des prélèvements sanguins ont été effectués chez chaque joueur.

Les joueurs ont tous donné leur consentement éclairé et écrit pour participer à l'étude, qui a été réalisée selon les recommandations de la déclaration d'Helsinki (Association Médicale Mondiale, 1964) 
modifiées en 2013. L'approbation préalable du Comité Scientifique Sectoriel des Sciences et Techniques des Activités Physiques et Sportives (CSS/STAPS) de l'université d'Abomey-calavi a été requise.

\section{Participants}

L'étude a été entreprise avec 16 joueurs volontaires, dont 8 de la catégorie junior et 8 seniors, appartenant tous à l'élite du Bénin. Les joueurs ont été répartis de façon aléatoire en deux équipes $\mathrm{A}$ et $\mathrm{B}$ comprenant chacune quatre handballeurs juniors (JJ) et quatre seniors (JS) pour les matchs expérimentaux. Parmi eux, 10 joueurs de niveau international, ont été vice-champions du Challenge Trophy continental en 2017. Les joueurs retenus, mais absents à une séance de prise de mesures ou malades ou blessés au cours de la période de trois jours ont été exclus de l'échantillon d'étude.

\section{Mesures}

Chaque fois, $5 \mathrm{~mL}$ de sang ont été prélevés par tube chez tous les joueurs, par ponction veineuse au pli cubital du coude gauche, dans des tubes EDTA et secs. Le sang prélevé a été conservé à la température ambiante pendant moins de 2 heures, pour déterminer la numération leucocytaire (leucocytes totaux, polynucléaires neutrophiles, lymphocytes) avec un cytomètre XN550 2016 (Sysmex, Allemagne). Les moyennes usuelles utilisées par le laboratoire chargé des analyses dans le cadre de cette étude se présentent comme suit: leucocytes totaux de 3 à $8 \mathrm{G} / \mathrm{L}$; neutrophiles de 1,5 à 6 $\mathrm{G} / \mathrm{L}$; lymphocytes de 1,5 à $4 \mathrm{G} / \mathrm{L}$.

Le sang contenu dans les tubes secs a été centrifugé à $3000 \mathrm{tr} / \mathrm{min}$ pendant $10 \mathrm{mn}$ dans l'heure qui a suivi les prélèvements, pour récupérer les sérums conservés à $-80{ }^{\circ} \mathrm{C}$ et utilisés pour le dosage des cytokines IL-6, IL10 et TNF- $\alpha$. Les kits ELISA Ready-Set-toGo, Invitrogen ${ }^{\circledR}$ ont permis de déterminer les concentrations sériques de chaque cytokine, conformément au protocole du fournisseur. La technique ELISA en "Sandwich", permettant le dosage plus sensible des cytokines via des anticorps spécifiques de capture et de détection des cytokines a été couplée à une réaction enzymatique colorimétrique, proportionnelle à la concentration en cytokines dans le serum.

Les seuils de détection des cytokines étaient de $2 \mathrm{pg} / \mathrm{mL}$ pour IL-6 et IL-10, puis de $4 \mathrm{pg} / \mathrm{mL}$ pour le TNF- $\alpha$. L'appareil Thermo Scientific $^{\mathrm{TM}}$ Wellwash $^{\mathrm{TM}}$ versa microplate a été utilisé pour le lavage des plaques après chaque étape. A la fin de la réaction, les densités optiques du produit obtenu pour chaque cytokine ont été mesurées à $450 \mathrm{~nm}$ selon les recommandations du fournisseur, à l'aide du Tecan plate reader Infinite 200Pro ${ }^{\circledR}$ et du logiciel Tecan ${ }^{\circledR}$ i-control version 1.12.4.0. Les courbes de concentration obtenues à partir des standards fournis par le fabricant (IL-6 de 2 à 200 pg/mL, IL-10 de 2 à $300 \mathrm{pg} / \mathrm{mL}$ et TNF- $\alpha$ de 4 à $500 \mathrm{pg} / \mathrm{mL}$ ) ont permis la détermination de la concentration de chaque cytokine, en utilisant le logiciel excel ${ }^{\circledR} 2010$. Chaque joueur a pris son dernier repas plus de trois heures avant le début de ses prélèvements et s'est abstenu de tout effort physique pendant les 72 heures qui ont précédé le premier match.

La taille des joueurs a été mesurée au millimètre près, en utilisant une toise murale graduée 206 M (Seca-Bodymeter, France) et la masse corporelle à $100 \mathrm{~g}$ près, avec un pèse personne (Terraillon, Chine). Ils étaient tous vêtus uniquement d'une culote et d'un tee shirt léger. Des thermomètres automatiques MT 101R (Hangzou Sejoy, Chine) ont été utilisés pour mesurer la température rectale. La fréquence cardiaque a été mesurée au repos (Fcr) et en fin de match avec des cardiofréquencemètres FT4 (Polar, Finlande). Un lecteur de bande DVD/CD et une clé USB préenregistrée du test $30-15$ IFT ont servi à 
estimer le $\dot{V} \mathrm{O}_{2} \max _{30-15}$ IFT de façon indirecte sur le terrain. L'indice physiologique de contrainte thermique (IPCT) a été calculé selon la formule: IPCT $=5\left(\mathrm{~T}_{\text {ract }}-\mathrm{T}_{\text {re } 0}\right) \mathrm{x}$ $\left(39,5-\mathrm{T}_{\mathrm{re} 0}\right)^{-1}+5\left(\mathrm{Ha}_{\mathrm{rt}}-\mathrm{HR}_{0}\right) \times\left(180-\mathrm{HR}_{0}\right)^{-1}$, avec $\mathrm{T}_{\text {reco } 0}$ et $\mathrm{T}_{\text {ract }}$ représentant la température rectale avant et après l'épreuve, $\mathrm{HR}_{0}$ et $\mathrm{Ha}_{\mathrm{rt}}$ la fréquence cardiaque avant et après l'épreuve (Moran et al., 1998). L'échelle d'appréciation de L'IPCT proposée par ces auteurs, a été utilisée pour déterminer le niveau de l'astreinte thermique vécue par les joueurs au cours des matchs. L'eau bue a été évaluée juste à la fin de chaque match, sur la base des contenus des bouteilles individuelles de 1,5 L. La température ambiante et l'humidité relative ont été enregistrées au cours de chaque match, par un appareil multifonction automatique (Thermo Pro, France).

\section{Intervention}

Les trois matchs se sont déroulés à Porto-Novo, en temps réglementaire de 30 $\min \times 2$ et une pause de $10 \mathrm{~min}$ (IHF, 2016), avec des arbitres qualifiés, entre 16 heures et 17 heures $30 \mathrm{~min}$ dans la soirée et étaient séparés de 24 heures de repos. Ils ont été joués en plein air, à une température ambiante variant de $30{ }^{\circ} \mathrm{C}$ à $32{ }^{\circ} \mathrm{C}$ et une humidité relative de $79 \%$ à $88 \%$. Pour limiter les pertes de temps, le chronométreur disposait d'une dizaine de balles qu'il pouvait introduire très rapidement sur le terrain à la demande des arbitres, en cas de sortie à la touche. Chaque joueur était équipé d'un cardiofréquencemètre protégé par un brassard en coton.

\section{Analyse statistique}

Les données ont été traitées avec le logiciel Satistica (Stat Soft Inc., version 12). Les résultats descriptifs ont été présentés sous forme de moyennes $(\mathrm{m}) \pm$ écart-type (s) pour les caractéristiques des handballeurs étudiés et de moyennes $(\mathrm{m}) \pm$ erreur standard de la moyenne pour les variables à l'étude. La normalité de la distribution des variables a été vérifiée en utilisant le test de KolmogorovSmirnov. En cas de distribution normale, une analyse de variance (ANOVA) à un facteur a été utilisée pour comparer les moyennes entre les trois temps de mesures, c'est-à-dire avant, à la fin et après 24 heures de récupération. Lorsque l'ANOVA était significative, le test post hoc de Tukey a été utilisé pour comparer les moyennes deux à deux. Le test $\mathrm{t}$ de Student pour échantillons indépendants a été utilisé pour comparer les deux catégories d'âges des seniors et des juniors entre elles. Lorsque la distribution n'était pas normale, le test de Friedman a été utilisé pour comparer les moyennes des trois temps de mesures. Quand le test de Friedman était significatif, le test de rang de Wilcoxon a été réalisé pour comparer les moyennes deux à deux. Le test $\mathrm{U}$ de Mann Whitney a été utilisé pour comparer les catégories d'âges entre elles. Le niveau de signification des tests statistiques a été fixé à $\mathrm{p}$ $<0,05$.

\section{RESULTATS}

Les moyennes respectives de la Fcr et $\mathrm{du} \stackrel{\mathrm{VO}}{2}_{2} \mathrm{max}$ des joueurs étaient de $56 \pm 1 \mathrm{bpm}$ et de $54,7 \pm 0,6 \mathrm{~mL} / \mathrm{min} / \mathrm{kg}$. Seuls l'âge et l'IMC avaient présenté des différences significatives $(p<0,05)$ entre les juniors et les seniors (Tableau 1).

$\mathrm{Au}$ cours de la série de matchs (Tableau 2), la température rectale a augmenté à la fin de tous les matchs $(\mathrm{p}<0,001)$ sans atteindre $39,5{ }^{\circ} \mathrm{C}$, puis sans différence significative entre le premier et le troisième match $(p>0,05)$. Aucun joueur n'a présenté au départ une Trec $\geq 38{ }^{\circ} \mathrm{C}$. A la fin de chaque match, la Fc a augmenté ( $\mathrm{p}<0,001)$, sans atteindre la Fcmax et sans que les différences entre le premier et le troisième match ne soient significatives $(p>0,05)$.

A la fin du premier match (Tableau 2), les moyennes de l'IL-6, du TNF- $\alpha$ et de l'IL10 se sont accrues respectivement de $232,0 \%$ 
$(\mathrm{p}<0,001), 566,1 \%(\mathrm{p}<0,001)$ et $266,6 \%$ (p $<0,001)$. Les tendances aux augmentations de $8,3 \%$ des leucocytes totaux, de $15,3 \%$ des neutrophiles et de baisse de $2,6 \%$ des lymphocytes à la fin du premier match n'étaient pas significatives $(p>0,05)$. A la fin du $3^{\text {e }}$ match par contre, les leucocytes totaux, les neutrophiles se sont accrus respectivement de $26,9 \%(p=0,01)$ et de $52,6 \%(p=0,002)$. Il en était de même pour l'IL-6 (282,7\%; $\mathrm{p}<$ $0,001)$, le TNF- $\alpha(626,6 \% ; p<0,001)$ et l'IL$10(118,4 \% ; p<0,001)$.

Les accroissements des moyennes des leucocytes totaux, des neutrophiles, de l'IL-6, de 1'IL-10 et du TNF- $\alpha$ enregistrés au cours $\mathrm{du}$ troisième match étaient plus importants $(\mathrm{p}$ $<0,05)$ que ceux observés à la fin du premier. Après 24 heures de récupération, les leucocytes totaux, les neutrophiles, ainsi que les lymphocytes ont été respectivement restaurés à $76,6 \%, 57,7 \%$ et $83,3 \%$ de leur valeur initiale, les différences de $23,4 \%$, $42,3 \%$ et $16,7 \%$ avec les valeurs initiales étant significatives $(\mathrm{p}<0,01)$. Par contre, les cytokines IL-6, TNF- $\alpha$, puis IL-10 étaient encore demeurés respectivement à $136 \%$, $138,7 \%$ et $142,8 \%$ au-dessus de leurs valeurs initiales, les différences de $36,0 \%, 38,7 \%$ et $42,8 \%$ étant toutes significatives ( $p<0,01$ ).

Le pourcentage de la Fcmax atteint par les joueurs, ainsi que l'IPCT (Tableau 3) n'ont pas varié significativement au cours des trois matchs $(p>0,05)$. La quantité d'eau bue par les handballeurs étudiés a été la plus élevée au cours du troisième match $(p<0,01)$.

Tableau 1 : Caractéristiques anthropométriques, physiologiques et antécédents de pratique sportive des handballeurs étudiés.

\begin{tabular}{|c|c|c|c|}
\hline & $\begin{array}{l}\text { Seniors } \\
(n=8)\end{array}$ & $\begin{array}{l}\text { Juniors } \\
(n=8)\end{array}$ & $\begin{array}{c}\text { Echantillon entier } \\
(n=16)\end{array}$ \\
\hline Age (ans) & $26,5 \pm 3,5$ & $18,8 \pm 1,1 * * *$ & $22,6 \pm 4,6$ \\
\hline Taille (cm) & $177,6 \pm 6,2$ & $177,4 \pm 6,6$ & $177,5 \pm 6,2$ \\
\hline Indice de masse corporelle $\left(\mathrm{kg} / \mathrm{m}^{2}\right)$ & $25,0 \pm 3,0$ & $22,4 \pm 2,4^{*}$ & $23,7 \pm 2,9$ \\
\hline Ancienneté dans la pratique (ans) & $11,7 \pm 1,6$ & $10,3 \pm 1,3$ & $11,0 \pm 1,6$ \\
\hline MHE (heures) & $6,7 \pm 1,0$ & $7,0 \pm 1,0$ & $6,8 \pm 1,0$ \\
\hline$\dot{\mathrm{VO}}_{2} \max _{30-15 \mathrm{IFT}}(\mathrm{mL} / \mathrm{min} / \mathrm{kg})$ & $55,0 \pm 2,3$ & $54,4 \pm 2,6$ & $54,7 \pm 2,4$ \\
\hline Fcr (bpm) & $56 \pm 4$ & $56 \pm 3$ & $56 \pm 3$ \\
\hline
\end{tabular}


Tableau 2 : Modifications des paramètres anthropométriques, physiologiques et de la réponse inflammatoire en match chez les handballeurs ( $\mathrm{n}=16$ ).

\begin{tabular}{|c|c|c|c|c|c|c|c|c|}
\hline & \multicolumn{3}{|c|}{$\mathbf{1}^{\mathrm{er}}$ Match } & \multirow{2}{*}{$\begin{array}{l}2^{\mathrm{e}} \text { Match } \\
\text { Avant }^{\alpha}\end{array}$} & \multicolumn{2}{|c|}{$3^{\mathrm{e}}$ Match } & \multirow[b]{2}{*}{$\Delta \mathbf{M} 2$} & \multirow[b]{2}{*}{$24 \mathrm{H}$ après } \\
\hline & Avant & Fin & $\Delta \mathbf{M 1}$ & & Avant & Fin & & \\
\hline Leucocytes T (G/L) & $6,0 \pm 0,3$ & $6,5 \pm 0,4$ & $+0,5$ & $5,5 \pm 0,2$ & $5,2 \pm 0,2$ & $6,6 \pm 0,4^{* *}$ & $+1,4^{\dagger \dagger}$ & $4,6 \pm 0,2^{\$ \$ \$}$ \\
\hline Neutrophiles (G/L) & $2,6 \pm 0,1$ & $3,0 \pm 0,3$ & $+0,4$ & $2,7 \pm 0,2$ & $1,9 \pm 0,1$ & $2,9 \pm 0,3^{* *}$ & $+1,0^{\dagger}$ & $1,5 \pm 0,1^{\$ \$ \$}$ \\
\hline Lymphocytes (G/L) & $3,0 \pm 0,1$ & $2,9 \pm 0,3$ & $-0,9$ & $2,3 \pm 0,1$ & $2,7 \pm 0,1$ & $3,0 \pm 0,3$ & $+0,3$ & $2,5 \pm 0,1^{\$ \$}$ \\
\hline $\mathrm{TNF}-\alpha(\mathrm{pg} / \mathrm{L})$ & $6,2 \pm 0,5$ & $41,3 \pm 2,9^{* * *}$ & $+35,1$ & $7,0 \pm 0,6$ & $7,5 \pm 0,5$ & $54,5 \pm 2,7^{* * *}$ & $+47,0^{\dagger \dagger \dagger}$ & $8,6 \pm 0,7^{\$ \$ \$}$ \\
\hline IL-6 (pg/L) & $2,5 \pm 0,2$ & $8,3 \pm 0,5^{* * *}$ & $+5,8$ & $2,7 \pm 0,2$ & $2,9 \pm 0,2$ & $11,1 \pm 0,9^{* * *}$ & $+8,2^{\dagger}$ & $3,4 \pm 0,2^{\$ \$ \$}$ \\
\hline IL-10 (pg/L) & $2,1 \pm 0,1$ & $7,7 \pm 0,6^{* * *}$ & $+5,6$ & $2,3 \pm 0,2$ & $2,5 \pm 0,1$ & $32,1 \pm 2,6^{* * *}$ & $+29,6 \mathrm{ti \dagger}$ & $3,0 \pm 0,3^{\$ \$}$ \\
\hline $\operatorname{Trec}\left({ }^{\circ} \mathrm{C}\right)$ & $37,2 \pm 0,1$ & $38,4 \pm 0,0^{* * *}$ & $+1,2$ & $37,2 \pm 0,0$ & $37,1 \pm 0,0$ & $38,5 \pm 0,1^{* * *}$ & $+1,4$ & $37,1 \pm 0,0$ \\
\hline $\mathrm{Fc}(\mathrm{bpm})$ & $56 \pm 1$ & $145 \pm 2^{* * *}$ & +89 & $55 \pm 1$ & $57 \pm 1$ & $145 \pm 2^{* * *}$ & +88 & $57 \pm 3$ \\
\hline
\end{tabular}

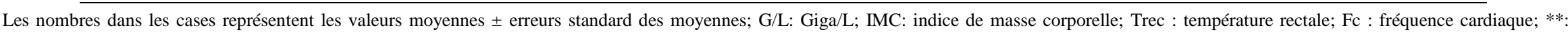

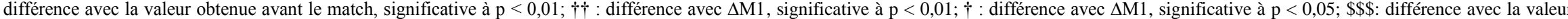

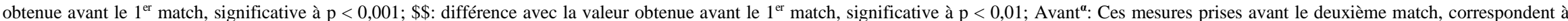
celles de la fin de la période de récupération de 24 heures après la fin du $1^{\text {er }}$ match.

Tableau 3 : Modifications des données de matchs chez les handballeurs étudiés $(\mathrm{n}=16)$.

\begin{tabular}{llll}
\hline & $\mathbf{1}^{\text {er }}$ Match & $\mathbf{2}^{\mathbf{e}}$ Match & $\mathbf{3}^{\mathrm{e}}$ Match \\
\hline Fcmax (bmp) & $188 \pm 2$ & $187 \pm 3$ & $189 \pm 2$ \\
Pourcentage de la Fcmax (\%) & $77,4 \pm 1,0$ & $77,1 \pm 0,9$ & $77,0 \pm 0,9$ \\
$\%$ de perte de poids (\%) & $+0,5 \pm 0,3$ & $-0,3 \pm 0,1^{*}$ & $+0,2 \pm 0,2$ \\
IPCT & $7,8 \pm 0,2$ & $7,8 \pm 0,2$ & $8,4 \pm 0,2$ \\
Quantité d'eau bue (L) & $1,3 \pm 0,1$ & $1,2 \pm 0,0$ & $2,4 \pm 0,1^{\dagger \dagger \$ \$}$
\end{tabular}

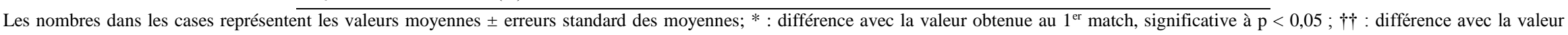

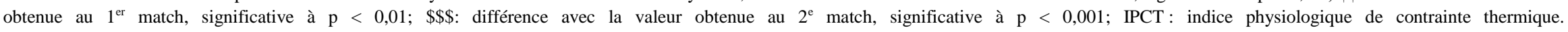




\section{DISCUSSION}

Cette étude a été entreprise sur la base des hypothèses selon lesquelles premièrement, les modifications des paramètres associés à la réponse inflammatoire observées à la fin du troisième match d'une série, sont plus importantes que celles observées à la fin du premier, chez les mêmes handballeurs. Deuxièmement, les valeurs des paramètres associés à la réponse inflammatoire ne sont pas totalement restaurées, 24 heures après la fin du troisième match de la série.

Les joueurs retenus pour entreprendre cette étude étaient suffisamment motivés pour participer aux différents travaux, laissant ainsi espérer un engagement total de leur part lors des matchs expérimentaux. Afin de garantir la fiabilité, voire la reproductibilité de la collecte de données, les matchs se sont déroulés dans les conditions réglementaires, avec des arbitres qualifiés et les méthodes utilisées pour le dosage des échantillons de sang étaient celles recommandées dans la littérature ou par les fabricants des réactifs. Toutefois, le fait que ces joueurs aient été sélectionnés par choix raisonné et donc par la méthode non probabiliste, rend les résultats valables pour les seuls handballeurs intégrés dans l'échantillon d'étude et non pour l'ensemble des joueurs du Bénin.

Les valeurs de la fréquence cardiaque de repos enregistrées aussi bien chez les juniors que chez les seniors dans cette étude, sont inférieures à $60 \mathrm{bpm}$ et témoignent d'un bon niveau de pratique de ces handballeurs, puisqu'elles correspondent aux valeurs usuelles rapportées dans la littérature chez les sportifs bien entraînés (Kenney et al., 2017).

Les valeurs du $\grave{V O}_{2} \max$ enregistrées chez les handballeurs de la présente étude sont comprises entre 50 et $60 \mathrm{~mL} / \mathrm{min} / \mathrm{kg}$, valeurs rapportées dans la littérature comme références de l'élite internationale du handball (Sporis et al., 2010). Il est donc permis de considérer que les 16 handballeurs impliqués dans cette étude sont d'un bon niveau de condition physique.

Bien qu'elle se soit accrue au cours de la série de matchs sans atteindre $39,5^{\circ} \mathrm{C}$, la température rectale n'a pas présenté de différence significative entre les trois matchs. Ce constat, ajouté à ceux relatifs aux pourcentages de la fréquence cardiaque maximale atteints, ainsi que les valeurs de l'indice physiologique de contrainte thermique qui n'ont pas varié, supportent l'hypothèse selon laquelle les joueurs se sont investis aux mêmes intensités au cours des trois matchs.

Au regard des conditions de réalisation de cette étude, c'est-à-dire avec des températures d'exposition souvent proches de $30{ }^{\circ} \mathrm{C}$ et une humidité relative atteignant $88 \%$, il fallait s'attendre à enregistrer des températures corporelles proches de $40{ }^{\circ} \mathrm{C}$ chez ces joueurs. Dans un tel contexte de pratique physique, la thermolyse devient en effet difficile et la température corporelle tend à s'élever rapidement (Banzet et al., 2012). Les quantités d'eau bue au cours des matchs ont certainement été suffisantes pour contribuer à éviter le phénomène d'hyperthermie corporelle chez ces joueurs (Banzet et al., 2012). Certains auteurs ont même rapporté qu'il est nécessaire d'optimiser la réhydratation de tous les athlètes par de l'eau enrichie en électrolytes (Kouassi et al., 2019).

A la fin du premier match, les moyennes de l'IL-6, du TNF- $\alpha$ et de l'IL-10 se sont accrues. Un résultat allant dans ce sens était attendu, car l'exercice physique intense induit une réponse inflammatoire médiée principalement par l'accroissement du taux des cytokines pro et anti-inflammatoires (Pérandini et al., 2016). Les cytokines proinflammatoires comme le TNF- $\alpha$ et l'IL-6 sont produites très tôt, en réponse aux efforts multiples et sont impliquées dans l'immunité tant innée qu'acquise (Nielsen et al., 2016). Ils se comportent comme des signaux intercellulaires qui règlent les réponses locales et systémiques. Par contre, les cytokines antiinflammatoires comme l'IL-10 ont la propriété d'une substance qui réduit et protège contre l'inflammation (Nielsen et al., 2016).

Les résultats de la présente étude par rapport au TNF- $\alpha$ et à l'Il-6 corroborent ceux 
rapportés dans une étude dont le but était de comparer les réponses inflammatoires entre joueurs et joueuses de football, de 48 heures après la fin d'un match officiel (Souglis et al., 2015). Ces auteurs ont rapporté que l'Il-6 et le TNF- $\alpha$ ont augmenté de 2 à 4 fois les valeurs de repos, avec un pic juste après le match. Au handball, une étude sur les cytokines et le stress oxydatif chez 14 joueurs brésiliens de l'élite, a également montré un accroissement du taux de l'Il-6 après le match (Marin et al., 2011). Il a été également rapporté comme dans la présente étude, une augmentation de l'IL-10 chez des athlètes, après un exercice intense et prolongé (Nielsen et al., 2016).

A la fin du premier match joué à une intensité relativement élevée, c'est-à-dire à $77,4 \%$ de la Fcmax, donc compris entre 70 et 90\% (sellami et al., 2018), des accroissements $\mathrm{du}$ nombre des leucocytes totaux, des neutrophiles et une réduction de ceux des lymphocytes étaient attendus. Les augmentations et baisses enregistrées n'ont pas été significatives. Au cours d'un exercice intense, une augmentation de la numération des leucocytes totaux, des neutrophiles, provoquée par le stress élevé (Bigley et Simpson, 2015), une lymphocytose médiée par la sécrétion des catécholamines (CuryBoaventura et al., 2018) s'observe souvent. Immédiatement après l'exercice ou au début de la récupération, l'effectif des leucocytes change et commence à retourner à la valeur de repos (Bigley et Simpson, 2015). Un second accroissement retardé des neutrophiles, dont la magnitude est liée à l'intensité et à la durée de l'exercice, est ensuite observé. Cet accroissement qui survient plus tard, peut être attribué au cortisol, dont la sécrétion induit la libération des neutrophiles dans la moelle osseuse (Pedersen et Febbraio, 2008). Sous l'effet du cortisol, la lymphocytose se transforme en lymphopénie, avant de remonter à la valeur de repos (CuryBoaventura et al., 2018). Il faut signaler que la production de cortisol est stimulée par l'IL-6, dont la concentration dépend fortement de l'intensité de l'exercice (Terra et al., 2012).
A la lumière de cette succession de phénomènes physiologiques qui se produisent pendant et après un exercice intense, il est possible de lier l'absence d'augmentation significative du nombre de leucocytes totaux, des neutrophiles et la réduction des lymphocytes à l'intensité à laquelle les handballeurs ont joué le premier match. Il faut en effet faire remarquer que l'intensité moyenne de $77 \%$ Fcmax atteinte dans ce match, est bien plus faible que les $85 \%$ Fcmax auxquels les matchs de haut niveau sont joués au handball (Póvoas et al., 2012). Il est donc possible que cette intensité n'ait pas été suffisante pour provoquer une forte production de cytokine IL-6. Cela a probablement réduit l'effet stimulant du cortisol, influençant ainsi l'ampleur du second accroissement retardé des neutrophiles, ainsi que celle de la baisse passagère de l'effectif des lymphocytes. Cette hypothèse est soutenue par les résultats de l'étude qui a rapporté une corrélation significative entre l'augmentation du taux de cortisol et celle de l'effectif des neutrophiles, après deux matchs consécutifs de football (Malm et al., 2004).

Le nombre des leucocytes totaux, des neutrophiles et les concentrations de l'IL-6, du TNF- $\alpha$ et de l'IL-10 se sont accrus à la fin du troisième match. Cette augmentation n'est pas liée à la seule intensité du troisième match. L'accumulation des charges physiques associées à l'effort physique fourni par les joueurs au cours des trois matchs a pu intervenir également. Le constat selon lequel l'accroissement du nombre des leucocytes totaux, des neutrophiles, des concentrations de l'IL-6, de l'IL-10 et du TNF- $\alpha$ enregistrées au cours du troisième match est plus important que celui observé à la fin du premier, rend compte de l'effet cumulé de la série de trois matchs sur ces cinq paramètres.

Ce résultat est d'autant plus normal que l'exercice physique intense répété sans une récupération suffisante, induit des lésions musculaires, accompagnées de libération accrue de cytokines TNF- $\alpha$, et IL-1 $\beta$. L'IL-6, cytokine sensible à l'inflammation, est ensuite produite (Pedersen et Febbraio, 2008). 
Cependant, cette IL-6, présente une action anti-inflammatoire indirecte, par la stimulation de la synthèse de l'IL-1ra et de l'IL-10 (Pedersen et Febbraio, 2008).

Les 24 heures de récupération prévues entre les matchs dans cette étude n'ont probablement pas été suffisantes pour réparer les lésions musculaires et résorber le processus inflammatoire mis en place par la charge physique imposée à l'organisme au cours de chaque match. En période de récupération, c'est sans doute cette continuité $\mathrm{du}$ processus inflammatoire et de lésions musculaires non résorbées, qui est à l'origine de l'important accroissement du nombre de leucocytes totaux, des neutrophiles, des taux de l'IL-6, puis du TNF- $\alpha$, provoquant ainsi l'élévation de l'IL-10, cytokine antiinflammatoire.

Après 24 heures de récupération, ce qu'il reste à restaurer pour les leucocytes totaux, les neutrophiles, les lymphocytes, les cytokines IL-6, TNF- $\alpha$ et IL-10 par rapport à leur valeur initiale était important. Les résultats suggèrent qu'il faut plus de 24 heures pour la restauration totale en ce qui concerne les marqueurs de la réponse inflammatoire, après une série de trois matchs joués par ces handballeurs du Bénin. D'autres auteurs ont également constaté que $24 \mathrm{~h}$ de récupération sont insuffisantes après un match de football pour assurer la récupération complète des marqueurs de dommage musculaire, puis de l'inflammation et qu'il fallait près de quatre jours pour que cela advienne (Morh et al., 2016).

Il est donc nécessaire que pendant la période de préparation aux compétitions, les entraîneurs proposent aux joueurs, des stratégies de récupération rapide de la fonction immunitaire. L'utilisation des méthodes d'entraînement qui obligent le joueur à travailler à des intensités proches de celles enregistrées pendant les matchs et la simulation des formules de compétitions sont entre autres, des stratégies possibles. Ce type d'entraînement et cette simulation vont sans doute favoriser une bonne adaptation des athlètes, permettant la récupération rapide des paramètres associés à la réponse inflammatoire (Khan et al., 2016). Une hydratation et des apports nutritionnels adéquats sont aussi d'une importance capitale dans la récupération rapide du système immunitaire (Walsh, 2019). En effet, les antioxydants tels que les vitamines $\mathrm{C}$ et $\mathrm{E}$, ainsi que les micronutriments comme le fer, le zinc et le magnésium qui jouent des rôles importants dans la synthèse de l'acide nucléique, limitent les dommages des tissus. C'est pour cela que la consommation d'antioxydants sous forme naturelle dans l'alimentation quotidienne, peut être d'une importance capitale, quand le stress oxydatif augmente avec la répétition des séances d'exercice physique d'intensités élevées. Il faut donc envisager une surveillance alimentaire chez ces joueurs et l'intégration d'aliments à forte teneur d'antioxydant dans leur régime.

\section{Conclusion}

Cette étude avait pour objectif de déterminer chez des handballeurs juniors et seniors appartenant à l'élite masculine du Bénin, les modifications des paramètres associés à la réponse inflammatoire au cours d'une série de trois matchs, ainsi que les taux de restauration de ces paramètres, atteints après 24 heures de récupération. Il est apparu que: 1) les accroissements des moyennes des leucocytes totaux, des neutrophiles, de l'IL-6, de l'IL-10 et du TNF- $\alpha$ enregistrés au cours $\mathrm{du}$ troisième match étaient plus importants que ceux observés à la fin du premier; 2) après 24 heures de récupération, les leucocytes totaux, les neutrophiles, les lymphocytes, ainsi que les cytokines IL-6, TNF- $\alpha$ et IL-10, ne sont pas restaurés à $100 \%$ de leurs valeurs initiales. Les résultats confirment l'hypothèse selon laquelle, la réponse inflammatoire observée chez des handballeurs de l'élite masculine à la fin du troisième match d'une série, est plus marquée que celle enregistrée à la fin du premier. Ils valident également l'hypothèse que les valeurs des paramètres étudiés ne sont pas totalement restaurées 24 heures après une série de trois matchs, ce qui 
pourrait compromettre la fonction immunitaire de ces joueurs. Si le temps de récupération n'est que de 24 heures, les entraîneurs devront proposer aux joueurs, des stratégies de récupération rapide pendant la période de préparation aux compétitions.

\section{CONFLIT D'INTERETS}

Les auteurs déclarent ne pas avoir de conflit d'intérêt.

\section{CONTRIBUTIONS DES AUTEURS}

LSOL: Elaboration du projet de recherche, collecte de données, traitement statistique, analyse et interprétations des données, rédaction du manuscrit; GP : validation $\mathrm{du}$ projet recherche, traitement statistique, analyse et interprétations des données, relecture et correction du manuscrit ; HT : validation du projet recherche, analyse et interprétations des données; MF : validation $\mathrm{du}$ projet recherche, traitement statistique; NBK : validation du projet recherche, collecte de données; KJP: traitement statistique, analyse et interprétations des données ; BA : validation du projet recherche, analyse et interprétations des données, relecture et correction du manuscrit.

\section{REMERCIEMENTS}

Les auteurs remercient tous les joueurs, entraîneurs et dirigeants des clubs de handball qui ont accepté de participer à la présente étude, en sacrifiant de leur temps et de leur énergie.

\section{REFERENCES}

Hermassi S, Ingebrigtsen J, Schwesig R, Fieseler G, Delank KS, Chamari K. 2018. Effects of in- season short-term aerobic and high-intensity interval training program on repeated sprint ability and jump performance in handball players. J. Sports. Med. Phys. Fitness, 58(1-2): 50-6. DOI: 10.23736/S00224707.16.06770-0

Nielsen HG, Øktedalen O, Opstad P, Torstein Lyberg T. 2016. Plasma cytokine profiles in long-term strenuous exercise.
J. Sports. Med., 2016: 1-7. DOI: $10.1155 / 2016 / 7186137$

Noack M, Kolopp-Sarda MN. 2018. Cytokines et inflammation: physiologie, physiopathologie et utilisation thérapeutique. Rev. Francoph. Lab., 2018(499): 28-37. DOI: 10.1016/S1773035X (18) 30052-2

Souglis A, Bogdanis GC, Chryssanthopoulos C, Apostolidis N, Geladas ND. 2018. Time course of oxidative stress, inflammation and muscle damage markers for 5 days after a soccer match: effects of sex and playing position. $J$. Strength. Cond. Res., 32(7): 2045-54. DOI: 10.1519/JSC.0000000000002436

Cury-Boaventura MF, Gorjão R, de Moura NR, Santos VC, Bortolon JR, Murata GM, Borges LDS, Momesso CM, Dermargos A, Pithon-Curi TC, Hatanaka E. 2018. The effect of a competitive futsal match on $\mathrm{T}$ lymphocyte surface receptor signaling and functions. Front. Physiol., 9: 202-209. DOI: 10.3389/fphys.2018.00202

Bessa AL, Olivera VN, Agostini GG, Oliveira RJ, Oliveira AC, White GE, Wells GD, Teixeira DNS, Espindola FS. 2016. Exercise intensity and recovery: biomarkers of injury, inflammation, and oxidative stress. J. Strength. Cond. Res., 30(2): 311-9. DOI: 10.1519/JSC.0b013e318f1ee9

International Handball Federation (IHF). 2016. Rules of the game. Lausane.

Póvoas M, Susana CA, Seabra R, André FT, Ascensão A, António A et al. 2012. Physical and physiological demands of elite team handball. J. Strength. Cond. Res., 26(12): 3365-75. DOI: 10.1519/JSC.0b013e318248aeee

Nouatin BK, Gouthon P, Amousou-Guénou D, Dansou PH, Messan F, Falola J-M, Tonon BA, Bio Nigan I, Arèmou M. 2015. Changes and recovery in fitness capacities following a series of handball matches played in the sub-tropical climate by division 1 amateur players. 
JBE., 12(2): 1-14. DOI: $10.4127 /$ jbe. 2015.0089

Pedersen BK, Hoffman-Goetz L. 2000. Exercise and the immune system: regulation, integration, and adaptation. Physiol. Rev., 80(3): 1055-81. DOI: 10.1152/physrev.2000.80.3.1055.

Harnish CR, Sabo RT. 2016. Comparison of two different sprint interval training work-to-rest ratios on acute inflammatory responses. Sports. Med. Open., 2: 20-8. DOI: 10.1186/s40798016-0044-1

Mohr M, Draganidis D, Chatzinikolaou A, Barbero-Alvarez JC, Castagna C. 2016. Muscle damage, inflammatory, immune and performance responses to three football games in 1 week in competitive male players. Eur. J. Appl. Physiol., 116(1): 179-93. DOI: $10.1007 / \mathrm{s} 00421-$ 015-3245-2

Gunzer W, Konrad M, Pail E. 2012. Exerciseinduced immunodepression in endurance athletes and nutritional intervention with carbohydrate, protein and fat - what is possible, what is not? Nutrients., 4(9): 1187-212. DOI: 10.3390/nu4091187

Gouthon P, Falola JM, Arèmou M, Koussihouèdé G, Anani L, Dansou P. 2009. Variations of the circulating leukocyte counts according to the volleyball players' practice level in Benin Republic. Rev. CAMES. Série A., 09: 549.

Asseh EE, Ake-Assi E, Koffi KJ. 2019. Diversité biologique et influence des changements climatiques sur la distribution géographique de quelques espèces d'Acanthaceae en Côte d'Ivoire. Int. J. Biol. Chem. Sci., 13(2): 676-692. DOI: $10.4314 /$ ijbcs.v13i2.9

Kouakou KJ, Gogbeu SJ, Sika AE, Yao KB, Bounakhla M, Zahry F. 2019. Caractérisation physico-chimique des horizons de surface de sols à maraîchers dans la ville d'Abidjan (Côte d'Ivoire). Int. J. Biol. Chem. Sci., 13(2):1193-1200. DOI: $10.4314 /$ ijbcs.v13i2.47
Mitchell JB, Dugas JP, McFarlin BK, Nelson MJ. 2002. Effect of exercise, heat stress, and hydration on immune cell number and function. Med. Sci. Sports. Exerc., 34(12): $\quad$ 1941-50. DOI: 10.1097/00005768-200212000-00013.

Buchheit M. Le 30-15 intermittent fitness test. 2005. Approches du Handball., 89: 42-7.

World Medical Association Declaration of Helsinki. 2013. Ethical principles for medical research involving human subjects. Adopted by the 18th WMA General Assembly, Helsinki, Finland, June 1964, and amended by the 64th WMA General Assembly, Fortaleza, Brazil, October 2013.

Moran DS, Shitzer A, Pandolf KB. 1998. A physiological strain index to evaluate heat stress. Am. J. Physiol., 275(44): 129-34.

Kenney L, Wilmore J, Costill D. 2017. Physiology of the sport and the exercise (6th edn). [Online]. Bruxelles: De Boeck University. Available: www.Deboeck superieur.com

Sporis G, Vuleta D, Dinko-Vuleta JR, Milanovic D. 2010. Fitness profiling in handball: physiological characteristics of elite players. Coll. Antropol., 34(3): 1009-14.

Banzet S, Koulmann N, Bourdon L. 2012. Activité physique et hyperthermie. Méd. Armées., 40(3): 207-16.

Kouassi JP, N'Guéssan K, Tonon B A, GOUTHON P, Nouatin BK, Tako A. 2019. Effet de la perte rapide volontaire de poids au cours d'un camp d'entraînement de deux semaines sur les paramètres hydro-électrolytiques et les performances des judokas de l'élite en côte d'Ivoire. Int. J. Biol. Chem. Sci., 13(7): $\quad 3119-30 . \quad$ DOI: https://dx.doi.org/10.4314/ijbcs.v13i7.12

Perandini LA, Sales-de-Oliveira D, Almeida DC, Azevedo H, Moreira-Filho CA, Cenedeze MA, Benatti FB, Lima FR, Borba E, Bonfa E, Sa-Pinto, Roschel H, Camara NOS, Gualano B. 2016. Effects of acute aerobic exercise on leukocyte 
inflammatory gene expression in systemic lupus erythematosus. Exerc. Immunol. Rev., 22: 64-81.

Souglis AG, Papapanagiotou A, Bogdanis GC, Travlos A, Apostolidis N, Geladas ND. 2015. Comparison of inflammatory responses to a soccer match between elite male and female players. $J$. Strength. Cond. Res., 29(5): 1227-33. DOI: 10.1519/JSC.000000 0000000767

Marin DP, Macedo dos Santos RC, Bolin AP, Guerra BA, Hatanaka E, Otton R. 2011. Cytokines and oxidative stress status following a handball game in elite male players. Oxid. Med. Cell. Longev., 2011: 1-10. DOI: $10.1155 / 2011 / 804873$

Sellami M, Gasmi M, Denham J, Hayes LD, Stratton D, Padulo J, Bragazzi N. 2018. Effects of acute and chronic exercise on immunological parameters in the elderly aged: Can physical activity counteract the effects of aging? Front. Immunol., 9: 2187-204.

DOI: 10.3389/fimmu.2018.02187

Bigley AB, Simpson RJ. 2015. NK cells and exercise: implications for cancer immunotherapy and survivorship. Discov. Med., 19(107): 433-45.

Pedersen BK, Febbraio MA. 2008. Muscle as an endocrine organ: focus on muscle- derived interleukin-6. Physiol. Rev., 88(4): $\quad 1379-406 . \quad$ DOI: 10.1152/physrev.90100.2007.

Terra R, da Silva SAG, Pinto VS, Dutra PML. 2012. Effect of exercise on the immune system : response, adaptation and cell signaling. Rev. Bras. Med. Esporte., 18(3): 208-14. DOI: $10.1590 / \mathrm{S} 1517-$ 86922012000300015

Malm C, Ekblom O, Ekblom B. 2004. Immune system alteration in response to two consecutive soccer games. Acta. Physiol. Scand., 180(2): 143-55. DOI: 10.1046/j.0001-6772.2003.01 232.x

Khan MA, Moiz AJ, Raza S, Verma S, Shareef MY, Anwer S, Ahmad A. 2016. Physical and balance performance following exercise induced muscle damage in male soccer players. J. Phys. Ther. Sci., 28(10): 2942-9. DOI: $10.1589 /$ jpts.28.3942

Walsh NP. 2019. Nutrition and athlete immune health: new perspectives on an old paradigm. Sports. Med. 49(2): 15368. DOI : 10.1007/s40279-019-01160-3. 